\title{
Crystal structures of NAC domains of human nascent polypeptide-associated complex (NAC) and its aNAC subunit
}

\author{
Lanfeng Wang ${ }^{1}$, Wenchi Zhang ${ }^{1}$, Lu Wang ${ }^{1}$, Xuejun C.Zhang ${ }^{1}$, Xuemei $\mathrm{Li}^{1}$, Zihe Rao ${ }^{1,2,3} \bowtie$ \\ ${ }^{1}$ National Laboratory of Biomacromolecules, Institute of Biophysics, Chinese Academy of Sciences, 15 Datun Road, Beijing \\ 100101, China \\ 2 Structure Biology Laboratory, Tsinghua University, Beijing 100084, China \\ 3 Tianjin Key Laboratory of Protein Science, College of Life Sciences, Nankai University, Tianjin 300071, China \\ $\triangle$ Correspondence: raozh@xtal.tsinghua.edu.cn \\ Received March 28, 2010 Accepted April 12, 2010
}

\begin{abstract}
Nascent polypeptide associated complex (NAC) and its two isolated subunits, $\alpha$ NAC and $\beta N A C$, play important roles in nascent peptide targeting. We determined a $1.9 \AA$ resolution crystal structure of the interaction core of NAC heterodimer and a $2.4 \AA$ resolution crystal structure of aNAC NAC domain homodimer. These structures provide detailed information of NAC heterodimerization and aNAC homodimerization. We found that the NAC domains of $\alpha$ NAC and $\beta N A C$ share very similar folding despite of their relative low identity of amino acid sequences. Furthermore, different electric charge distributions of the two subunits at the NAC interface provide an explanation to the observation that the heterodimer of NAC complex is more stable than the single subunit homodimer. In addition, we successfully built a $\beta$ NAC NAC domain homodimer model based on homologous modeling, suggesting that NAC domain dimerization is a general property of the NAC family. These 3D structures allow further studies on structurefunction relationship of NAC.
\end{abstract}

KEYWORDS nascent polypeptide-associated complex, aNAC homodimer, $\beta N A C$, crystal structure

\section{INTRODUCTION}

Nascent polypeptide associated complex (NAC) heterodimer of $\alpha \mathrm{NAC}$ (NACA) and $\beta N A C$ (BTF3b) is characterized as the first cytosolic factor that binds nascent polypeptides emerging from the ribosome and prevents the peptides from incorrectly binding with other cytosolic factors (Wiedmann et al., 1994). This complex is widely conserved from archaea to human. Some NAC mutants induce early embryonically lethal phenotypes in mice, fruit fly, and Caenorhabditis elegans (Deng and Behringer, 1995; Markesich et al., 2000). Experiments to determine NAC intracellular localization and distribution show that the vast majority of NAC is in the cytoplasm as a stable heterodimer, and no single subunit and homo-oligomer has been observed under physiologic conditions (Beatrix et al., 2000). However, drastic variations of relative concentration between the two NAC subunits are found in patients of Alzheimer's disease, Down syndrome, malignant brain tumors, AIDS, and ulcerative colitis (Zuo et al., 1997; Scheuring et al., 1998; Kroes et al., 2000; Kim et al., 2002), which indicates that imbalanced NAC subunits may result in pathological conditions. Sequence analysis predicts that the two NAC subunits share low yet recognizable amino acid sequence similarity in a region termed as the NAC domain. However, there is a UBA (ubiquitin-associated) domain, only in aNAC but not $\beta N A C, C$-terminal to the NAC domain, implicating different functions for the two subunits (Spreter et al., 2005). Saccharomyces cerevisiae genome encodes one aNAC homolog and two BNAC homologs (Shi et al., 1995; Reimann et al., 1999). In contrast, all sequenced archaebacterial genomes have only one gene, named as aeNAC, homologous to human aNAC (Spreter et al., 2005). Therefore, NAC is likely to be an ancient protein complex with some latter-acquired functions.

At present, it is widely accepted that NAC plays an important role in cotranslational targeting of nascent polypeptides to endoplasm reticulum (ER). Either of the two NAC subunits can bind directly with nascent polypeptides 
emerging from the ribosome, albeit the binding of aNAC is rather weak. $\beta N A C$ can directly bind ribosome protein near the exit site of nascent polypeptide using its $\mathrm{N}$-terminal ribosome binding motif (Wegrzyn et al., 2006). In contrast, aNAC does not bind ribosome protein directly. Nevertheless, it can interact with ribosome by binding tightly with ribosome nucleic acid in a non-sequence-specific manner (Beatrix et al., 2000). Moreover, the NAC complex can start binding nascent polypeptide when the $\mathrm{N}$ terminus of the peptide is only 17-residues long from the peptidyl-transferase center, and NAC starts being released after the polypeptide becomes longer than 30 residues. Protease digestion assays confirm the NAC protection for nascent polypeptide (Wang et al., 1995). The binding and releasing of NAC for nascent polypeptide appear in quantal units instead of a residue-byresidue manner. This binding is independent of signal peptide sequences, and yet NAC cooperates with signal recognition particle (SRP) for correctly targeting and folding of nascent polypeptide in the cotranslational process (Lauring et al., 1995a, b; Powers and Walter, 1996; Moller et al., 1998b). However, yeast NAC homolog (termed as EGD complex) is not prerequisite for growth (Reimann et al., 1999), but is necessary for protein targeting to mitochondria (George et al., 1998; Fünfschilling and Rospert, 1999). In addition, in yeast both subunits including their NAC domains have been shown to be ubiquitinated in a lysine dependent manner (Panasenko et al., 2006, 2009). Besides functioning in cytosol, NAC can tightly bind in nucleus with $\mathrm{X}$-junction in DNA replication forks and homolog recombinant regions (Whitby and Dixon, 2001). Therefore, the heterodimer of NAC appears to be multifunctional.

In the absence of a hetero-partner, for example when overexpression or downregulation of a single subunit, the remaining dominant subunit may have functions independent of nascent peptide binding (Thiede et al., 2001; Spreter et al., 2005). For instance, isolated aNAC is a multiple functional protein taking part in transcription regulation (Moreau et al., 1998; St-Arnaud, 1998; St-Arnaud and Quelo, 1998; Yotov etal., 1998), cell proliferation, and differentiation (Al-Shanti etal., 2004; Lopez et al., 2005; Al-Shanti and Aldahoodi, 2006). Similarly, isolated BNAC (also called BTF3b) was initially identified as an alternative splicing isoform of the basic transcription factor (BTF3a). However, $\beta N A C$ is transcriptionally inactive although it has the same ability as BTF3a to bind RNA polymerase II (Zheng et al., 1987, 1990). Structural basis of such distinct functions remains to be determined.

The crystal structure of aeNAC shows that it forms a homodimer via an interaction between the NAC domains (Spreter et al., 2005). However, until now there has been no experimental data on how human NAC heterodimer is made from aNAC and BNAC and what the structural difference is between NAC and its subunit homodimer. In this study we used the crystallography method to address these questions. We determined the crystal structure of NAC hetero-dimeric interaction core complex at $1.9 \AA$ resolution and the crystal structure of aNAC NAC domain homodimer at $2.4 \AA$ resolution. In addition, our structural analysis and model building suggested that $B N A C$ subunits can form a homodimer similar to the NAC heterodimer. These structures provide detailed information of NAC heterodimerization and an explanation to why NAC is more stable than an aNAC homodimer.

\section{RESULTS}

\section{Structure of human NAC complex}

We coexpressed and copurified the full length recombinant proteins of human aNAC (GenBank: AAX14393.1, residues 1-215) and BNAC (GenBank: AAH08062.1, residues 1-162) from $E$. coli. Solution study showed that $\alpha N A C$ and $B N A C$ recombinant proteins formed a stable heterodimer ( Supplemental Fig. 1A). NAC crystals of good diffraction quality appeared six months after setting up the crystallization drops. The reservoir solution was $0.1 \mathrm{M}$ sodium citrate $(\mathrm{pH} 5.6)$, $0.1 \mathrm{M} \mathrm{NaCl}$, and $12 \%(w / v)$ polyethylene glycol (PEG) 4000 . Regarding the prolonged crystallization time, we speculated that the protein sample was processed by some incidentally contaminated protease(s), which promoted the crystallization. Phases of the crystal structure were solved at $1.9 \AA$ resolution using the single wavelength anomalous diffraction (SAD) data collected from a potassium iodine derivative. Subsequently, it was found that the crystal structure contained only the NAC domains from both aNAC (residues 79-132, designated as $N A C_{\alpha}$ ) and $\beta N A C$ (residues 53-110, named as $N A C_{\beta}$ ) forming a heterodimer of dimensions of $30 \times 34 \times 45 \AA$ (Fig. 1A). The solvent content of the crystal form was estimated to be $63 \%$ $\left(V_{M}=3.3 \AA^{3} / \mathrm{Da}\right)$ with one such heterodimer per asymmetric unit. The crystal structure was refined to $R$ factor of 0.196 (and R-free of 0.245) with excellent geometry (Table 1). Taken together, we obtained the crystal structure of a stable core of the human NAC heterodimer.

In this complex, $\mathrm{NAC}_{\alpha}$ and $\mathrm{NAC}_{\beta}$ subunits shared a similar $V$-shaped structure. The topology of the heterodimer is shown in Fig. 1B. NAC $\alpha$ consisted of helix $\alpha 1$ and strands $\beta 1-\beta 6$; and $N^{\prime} C_{\beta}$ consisted of strands $\beta 1^{\prime}-\beta 6^{\prime}$, helices $\alpha 1^{\prime}$ and $\alpha 2$ ' (structural elements of $\mathrm{NAC}_{\beta}$ are denoted as primes hereafter). In $N A C_{\alpha}, \alpha 1, \beta 1, \beta 4$, and $\beta 5$ were arranged in one arm of the $V$-shaped structure, and $\beta 2, \beta 3$, and $\beta 6$ were arranged in the other arm. Similarly, in $\mathrm{NAC}_{\beta}, \beta 1^{\prime}, \beta 4^{\prime}$, and $\beta 5^{\prime}$ were assembled in one arm, and $\beta 2^{\prime}, \beta 3^{\prime}, \beta 6^{\prime}, \alpha 1^{\prime}$, and $\alpha 2^{\prime}$ were in the other arm. The two $V$-shaped structures packed with each other in a head-to-head manner to generate a 12-stranded $\beta$ barrel-like heterodimer $\left(\mathrm{NAC}_{\alpha}-\mathrm{NAC}_{\beta}\right)$ with six strands in each of the two major $\beta$-sheets (Fig. 1A). Structure based sequence alignment (Fig. 1C) showed that there is neither insertion nor deletion between the NAC domains of aNAC and $\beta N A C$ subunits. Although amino acid-sequence identity between the NAC domains of $N A C_{\alpha}$ and $N A C_{\beta}$ is low $(10 / 48$ 

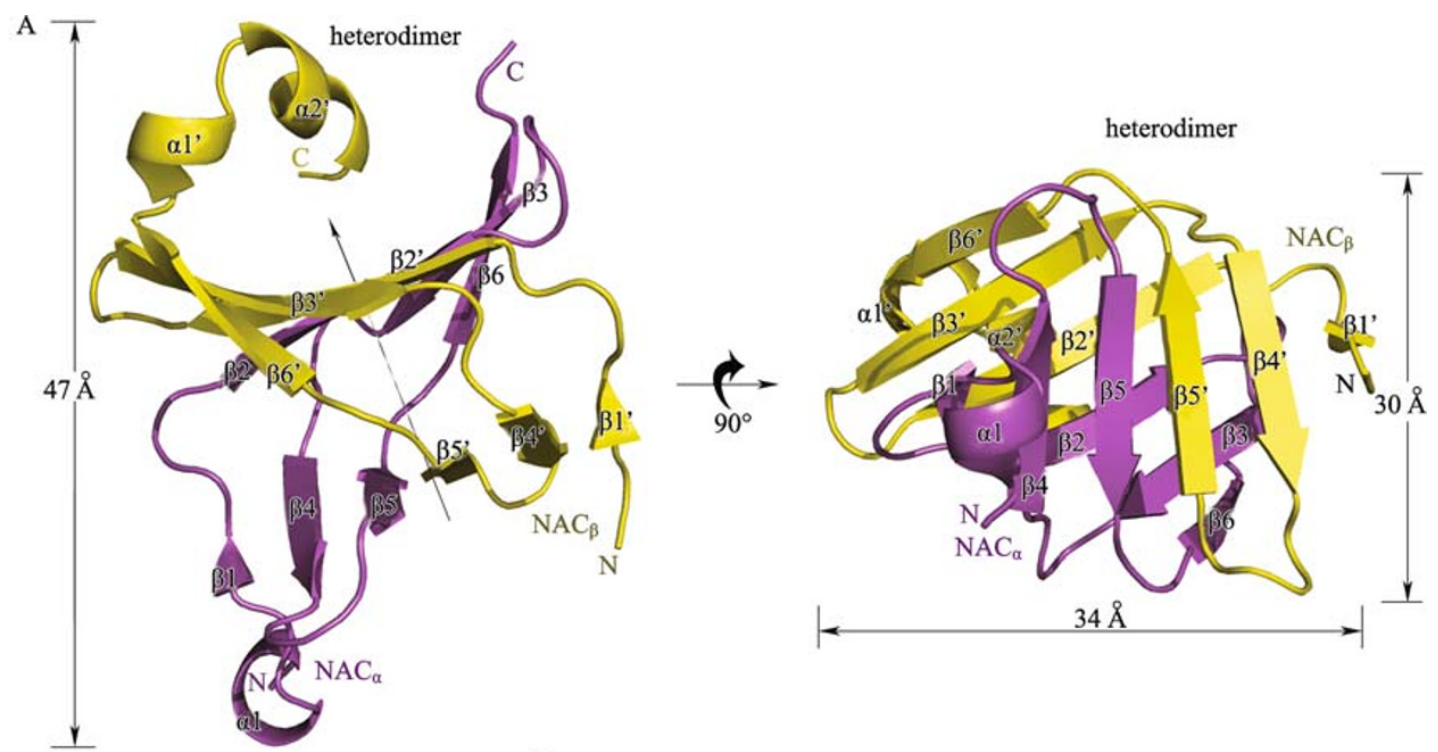

B

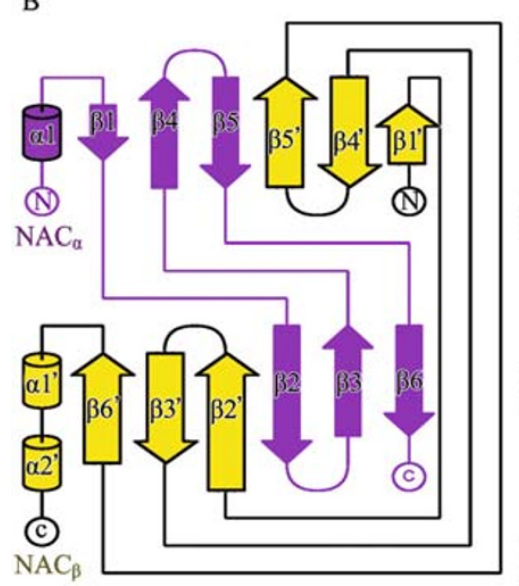

$\mathrm{C}$

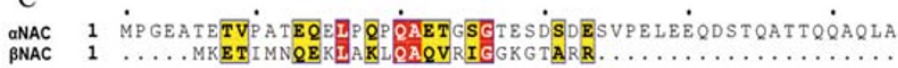

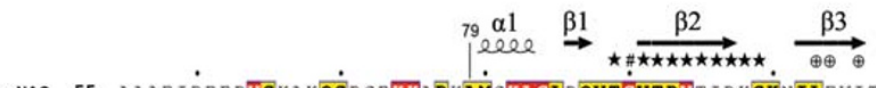

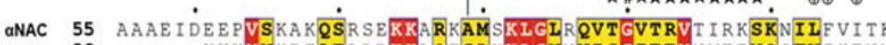
BNAC $29 \quad \ldots \ldots$ KKKVVHRTATADDKKLQFSIKKL GVNNISGIEEVNMFTNQGTVIHFNN

$$
\stackrel{\beta 4}{\underset{\oplus}{\longrightarrow}} \underset{\oplus \star \star \star \star \star \star \star \star \star \star \star \oplus \oplus \oplus . \oplus \mid}{\stackrel{\beta 5}{\longrightarrow}} \stackrel{\beta 6}{132}
$$

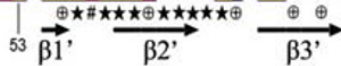

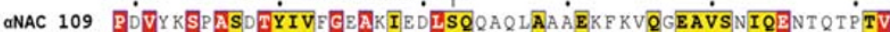
ANAC 77 BKVQASLAANTFTITGHAETKOLTEMLPSILNOLGADSLTSLRR LAEALPKOSV

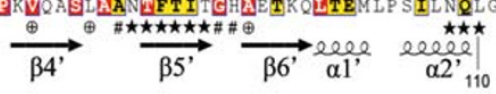

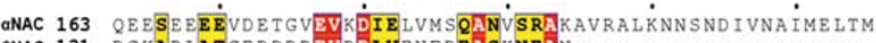

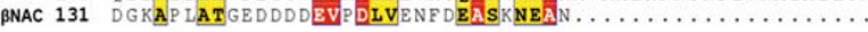
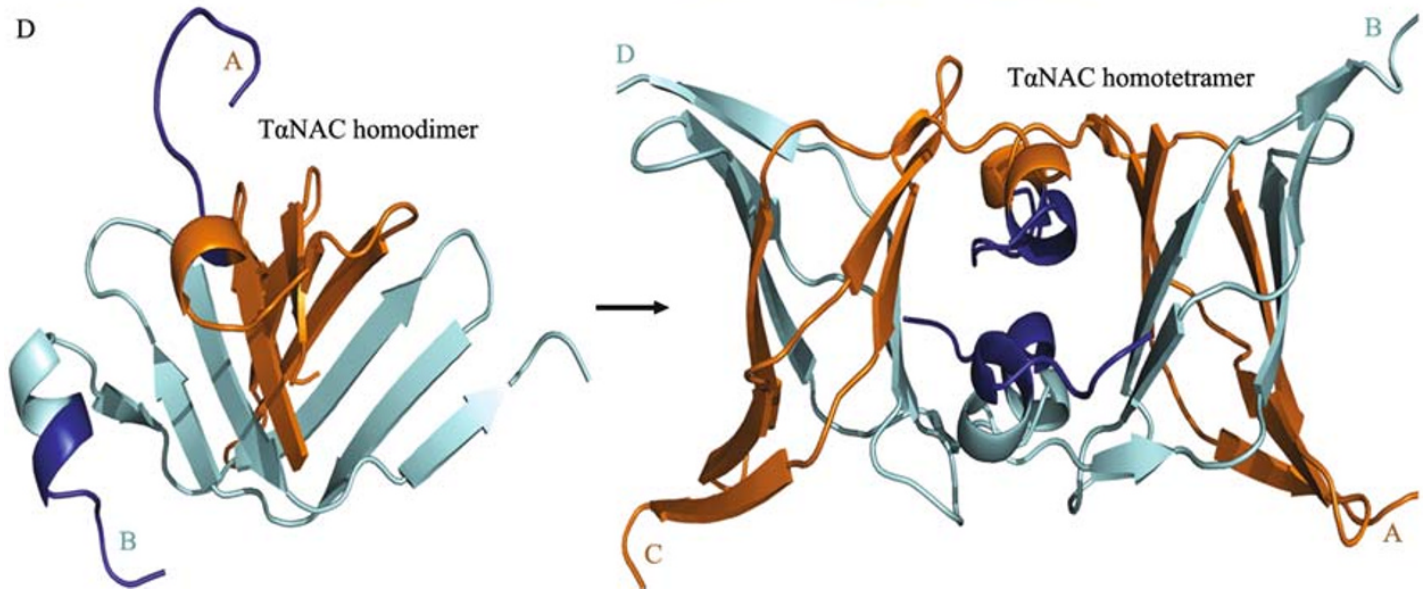

Figure 1. Structure of human NAC complex and TaNAC. (A) Ribbon diagram of the heterodimer $\mathrm{NAC}_{\alpha}-\mathrm{NAC}_{\beta}$ complex. NAC $\alpha$ (magenta) consists of an N-terminal helix (labeled as $\alpha 1$ ) and six $\beta$-strands ( $\beta 1-\beta 6)$. NAC $\beta$ (yellow) consists of six $\beta$-strands ( $\left.\beta 1^{\prime}-\beta 6^{\prime}\right)$ and two short helices ( $\alpha 1^{\prime}$ and $\alpha 2^{\prime}$ ) at the C-terminal. The pseudo dyad symmetry axis was marked as a black arrow in the left panel. (B) Topology of the $\mathrm{NAC}_{\alpha}-\mathrm{NAC}_{\beta}$ complex. $\alpha$-Helices were draw as cylinders, and $\beta$-strands were shown as arrows. Secondary structures are labeled and colored the same as panel A. (C) Sequence alignment of the two NAC subunits. Identical residues are highlighted with red color, and other conserved residues are highlighted with yellow color. Secondary structures of NAC and NAC $\beta$ were included on the top and at the bottom, respectively. Residues involved in heterodimerization were marked as following: \#, residues with only main chain atoms being involved in dimerization; $\star$, residues with both main chain atoms and side chain atoms being involved; and $\oplus$, residues with only side chain atoms being involved. Note that in the NAC domain region, this alignment is consistent with a 3D structure-based sequence alignment. (D) Ribbon presentation of the TaNAC homodimer and homotetramer. On the left side two protomers (chain $A$, orange; and chain $B$, cyan) form a homodimer; and on the right side two homodimers (AB and $\mathrm{CD} ; \mathrm{A}$ and $\mathrm{C}$ colored in orange; $\mathrm{B}$ and $\mathrm{D}$ in cyan) form a tetramer. The tetramer was formed mainly through $\mathrm{N}$-terminal 8 non-native residues (blue). 
Table 1 Data collection, SAD phasing and refinement statistics

\begin{tabular}{|c|c|c|}
\hline & TaNAC (KI derivative) & NAC complex (KI derivative) \\
\hline \multicolumn{3}{|l|}{ data collection } \\
\hline space group & $\mathrm{P} 2{ }_{1} 2{ }_{1} 2$ & P $6{ }_{5} 22$ \\
\hline \multicolumn{3}{|l|}{ cell dimensions } \\
\hline$a, b, c(\AA)$ & $54.6,59.6,69.1$ & $59.8,59.8,156.8$ \\
\hline$\alpha, \beta, \gamma\left({ }^{\circ}\right)$ & $90,90,90$ & $90,90,120$ \\
\hline wavelength & 1.5418 & 1.0000 \\
\hline resolution $(\AA)$ & $50-2.4(2.49-2.40)$ & 50-1.9 (1.97-1.90) \\
\hline$R_{\mathrm{sym}}$ or $R_{\text {merge }}$ & $0.076(0.282)$ & $0.050(0.533)$ \\
\hline$I / \sigma(\mathrm{I})$ & $39.5(9.1)$ & $42.3(3.98)$ \\
\hline completeness (\%) & $99.8(99.8)$ & $98.8(94.9)$ \\
\hline redundancy & $13.5(12.3)$ & $18.3(13.4)$ \\
\hline \multicolumn{3}{|l|}{ refinement } \\
\hline resolution $(\AA)$ & $29.9-2.4$ & $29.4-1.9$ \\
\hline No. reflections & 9151 & 12,225 \\
\hline$R_{\text {work }} / R_{\text {free }}$ & $0.242 / 0.275$ & $0.196 / 0.245$ \\
\hline \multicolumn{3}{|l|}{ No. atoms } \\
\hline protein & 1830 & 868 \\
\hline solvent & 43 & 48 \\
\hline \multicolumn{3}{|l|}{$B$-factors } \\
\hline protein & 51.1 & 52.6 \\
\hline solvent & 42.5 & 55.60 \\
\hline \multicolumn{3}{|l|}{ r.m.s deviations } \\
\hline bond lengths $(\AA)$ & 0.031 & 0.008 \\
\hline bond angles $\left({ }^{\circ}\right)$ & 1.90 & 1.12 \\
\hline \multicolumn{3}{|l|}{ ramachandran plot ${ }^{a}$} \\
\hline preferred region (\%) & 94.4 & 92.4 \\
\hline allowed region (\%) & 4.6 & 7.8 \\
\hline outlied region (\%) & 0.9 & 0.45 \\
\hline
\end{tabular}

${ }^{a}$ Calculated using the program Coot.

or $21 \%$ ), they shared very similar $3 \mathrm{D}$ folding. The rmsd (root mean square deviation) of $44 \mathrm{Ca}$ atom pairs was $1.05 \AA$ (using a $3.0 \AA$ cutoff, the same hereafter unless specified otherwise). There was a pseudo dyad symmetry in the heterodimer complex of $\mathrm{NAC}_{\alpha}-\mathrm{NAC}_{\beta}$ (Fig. 1A), and the corresponding rotation angle was $177^{\circ}$ with a minor screw length $(0.24 \AA)$ between the two subunits.

The dimerization of $\mathrm{NAC}_{\alpha}$ and $\mathrm{NAC}_{\beta}$ buried total of $2330 \AA^{2}$ solvent accessible surface from both subunits, corresponding to $36 \%$ of their total surface area. Interaction involved in the heterodimerization can be divided mainly into $\beta$-sheet formation contributed by main chain-main chain interaction and a hydrophobic core formed by side chain-side chain interaction. Residues of the first group were mainly distributed around the $\beta 2$ and $\beta 5$ strands (Fig. 1C), where one anti-parallel $\beta$-sheet was produced between $\beta 2$ and $\beta 2$,' and another one was generated by $\beta 5$ and $\beta 5$ '. There were total 15 intermolecular hydrogen bonds between $\mathrm{NAC}_{\alpha}$ and $\mathrm{NAC}_{\beta}$. Nine of them were around the two $\beta 2$ strands (Supplemental Table 1). It is worth to point out that there were a side chain-main chain hydrogen bond (NE/Arg93-O/ GIn109') and a side chain salt bridge (Nع/Arg97-Oع2/Glu61') in this group, which may be sensitive to mutations. In the main chain interface there was a bulge at Thr92 in the $\beta 2$ strand of $\mathrm{NAC}_{\alpha}$ (Supplemental Table 1). Because $\beta 2$ ' was shorter at the $\mathrm{N}$-terminal, this bulge was not conserved in $\beta N A C$. The rest six hydrogen bonds were around the two $\beta 5$ strands (Supplemental Table 1). Hydrophobic side chains buried inside the $\beta$-barrel of the dimer included Val91, Val94, lle96, Phe104, Ile106, Val111, Tyr120, Val122, Ala126, and Ile128 in molecule $\mathrm{NAC}_{\alpha}$ and Ile56', Ile59', Val62', Met64', Ile72', Phe74', Val79', Leu83', Phe88', Ile90', and Ala94' in molecule 
$\mathrm{NAC}_{\beta}$ (Fig. $1 \mathrm{C}$ and Supplemental Fig. 2). Taken together, the two NAC domains have an extensive interaction which appears essential for the stability of each subunit.

Despite of their folding similarity, the two subunits of NAC showed clear structural differences in a few regions. First, a short helix, $\alpha 1$, was located at the $\mathrm{N}$ terminus of $\mathrm{NAC}_{\alpha}$, while there were two short helices, $\alpha 1$ ' and $\alpha 2$ ', at the $C$ terminus of $\mathrm{NAC}_{\beta}$. Based on their high sequence similarity at $\mathrm{N}$-termini (Fig. 1C), we speculate that the reason why $\mathrm{NAC}_{\beta}$ crystal structure missed an $\mathrm{N}$-terminal short helix similar to that of $\mathrm{NAC}_{\alpha}$ is a truncation of the peptide instead of high mobility of the $\mathrm{N}$ terminus. In contrast, the region $\mathrm{C}$-terminal to $\mathrm{NAC}_{\alpha}$ shares no sequence homology with $\mathrm{NAC}_{\beta}$, and we do not expect similar secondary structures in the corresponding regions. Secondly, the lengths of $\beta 2$ and $\beta 2^{\prime}$, of $\beta 3$ and $\beta 3^{\prime}$, and of $\beta 4$ and $\beta 4$ ' varied between the two subunits. In addition, positions of $\beta 1$ and $\beta 1$ ' differed after overall superposition, and positions of the loops connecting $\beta 2$ and $\beta 3$ and connecting $\beta 4$ and $\beta 5$ strands also showed significant changes (Fig. 2A).

As shown in Fig. $1 \mathrm{~A}, \alpha 1^{\prime}$ and $\alpha 2$ ' of $\mathrm{NAC}_{\beta}$ were away from the NAC core. Structure inspection showed that some hydrophobic residues including Phe65', Val71', Leu99', Met102', Leu103', Ile106', Leu107', and Leu110' of NAC $\beta$ formed a small hydrophobic core between $\alpha 1^{\prime}, \alpha 2^{\prime}, \beta 2$ ', and $\beta 3^{\prime}$ (Supplemental Fig. 2A); additionally, Oع1/Gln109' of $\alpha 2$ ' formed a hydrogen bond with Nס2/Asn63' of $\beta 2$ ', and $\mathrm{O} /$ GIn109' of a2' could form another hydrogen bond with $N \varepsilon /$ Arg93 of $\mathrm{NAC}_{\alpha} \beta 2$ strand (Supplemental Fig. 2A). These intramolecular and intermolecular interactions likely contribute to the stability of helices $\alpha 1^{\prime}$ and $\alpha 2^{\prime}$ at the $\mathrm{NAC}_{\beta} \mathrm{C}$-terminus in the crystal and presumably in solution too.

\section{Structure of aNAC NAC domain homodimer}

It has been reported that $\alpha$ NAC may perform certain function in the absence of $\beta$ NAC (Thiede et al., 2001; Spreter et al., 2005). To study the structure of human aNAC alone, we constructed a number of aNAC variants of different lengths, including the full length and the NAC domain alone (i.e., residues $80-133$, named as TaNAC standing for truncated aNAC). In a solution study, size exclusion chromatography and analytical ultracentrifugation showed that both full length aNAC and TaNAC formed stable homodimers, separately (Supplemental Fig. 1B-E). While the recombinant proteins of full length and other constructs of aNAC did not give rise to crystals of high diffraction quality, TaNAC readily crystallized in $3-4 \mathrm{~d}$ in a hanging drop experiment using a reservoir solution of $0.2 \mathrm{M}$ potassium citrate $(\mathrm{pH} 8.3)$ and $20 \%(\mathrm{w} / \mathrm{v})$ PEG 3350. Similar to the above heterodimer crystal structure, the phases of the TaNAC crystal structure were determined at $2.4 \AA$ resolution using SAD data from a potassium iodine derivative. Although the solvent content of the TaNAC crystal $\left(41 \%\right.$ with $\left.\mathrm{V}_{\mathrm{M}}=2.1 \AA^{3} / \mathrm{Da}\right)$ was lower than that of $\mathrm{NAC}_{\alpha}-\mathrm{NAC}_{\beta}$ $(63 \%)$, the resolution of diffraction data used in refinement was lower for the TaNAC crystal $(2.4$ vs. $1.9 \AA)$. In fact, the TaNAC crystal indeed diffracted beyond $2 \AA$ resolution. However, shapes of the diffraction spots were problematic, and the R-merge value would be intolerably high if higher resolution data were included. Therefore, we chose to lower the resolution limit to $2.4 \AA$ during data processing.

The crystal structure consisted of four TaNAC protomers (chain A, residues 73-114 and 118-132; chain B, residues 74-133; chain $C$, residues $74-132$; and chain $D$, residues 77-133) per asymmetric unit (Fig. 1D). Structures of the four TaNAC protomers were similar to each other, and any two of them had an rmsd ranged from $0.6 \AA$ to $1.8 \AA$ (for all common Ca atoms, i.e., residues 77-114 and 118-132). Each TaNAC protomer (using molecule $A$ as a representative in the following discussion, the same hereafter unless otherwise specified) consisted of identical secondary structures as $\mathrm{NAC}_{\alpha}$ from the $\mathrm{NAC}_{\alpha}-\mathrm{NAC}_{\beta}$ heterodimer $(1.3 \AA \mathrm{rmsd}$ for $48 \mathrm{C} \alpha)$.

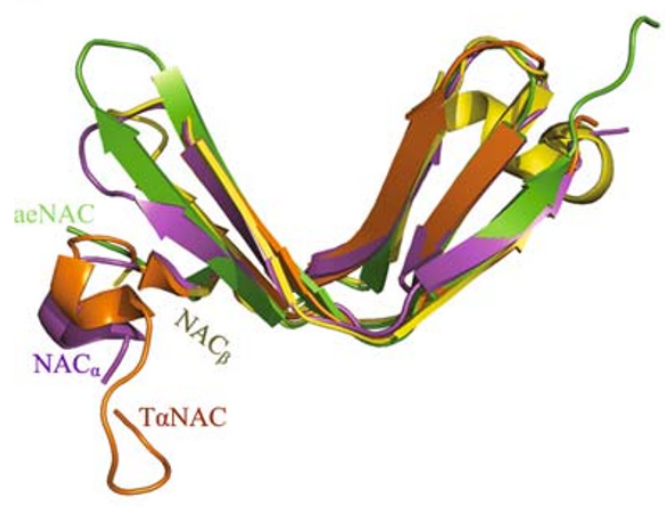

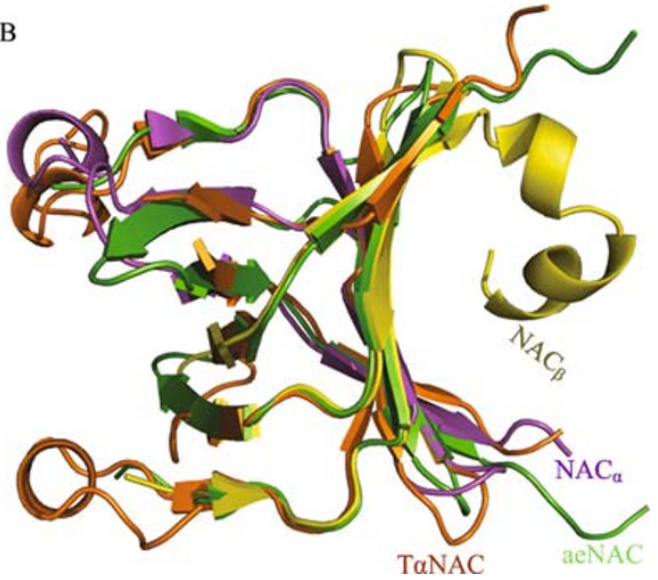

Figure 2. Superposition of homologous structures of human and archaea NAC domains. (A) NAC domain protomers including $\mathrm{NAC}_{\alpha}$ (magenta), $\mathrm{NAC}_{\beta}$ (yellow), TaNAC (orange), and aeNAC (green). (B) NAC $\mathrm{NAC}_{\beta}$ heterodimer, TaNAC homodimer, and aeNAC homodimer. 
The four TaNAC protomers formed two identical homodimers, denoted as $A B$ and $C D$, with an rmsd of $1.1 \AA$ for all 106 common Ca pairs. They were also similar to the $\mathrm{NAC}_{\alpha}{ }^{-}$ $\mathrm{NAC}_{\beta}$ heterodimer (1.1 A rmsd of 89 Ca pairs) (Fig. 2B). In the structure of TaNAC homodimer, stability of the TaNAC homodimer was contributed by total 14 intermolecular hydrogen bonds including a main chain-side chain one formed by O/Thr89/chain A and NC/Lys98/chain B (Supplemental Table 1) and by the interaction of hydrophobic side chains of Val88, Val91, Val94, Ile96, Ile102, Phe104, Ile106, Pro109, Val111, Tyr120, Val122, Ala126, and lle128 from both chains, which were buried inside the $\beta$-barrel (Supplemental Fig. 2B) just like the $\mathrm{NAC}_{\alpha}-\mathrm{NAC}_{\beta}$ heterodimer.

Furthermore, two such homodimers formed a tetramer in the crystal mainly through 8 non-native residues (i.e., ${ }^{73}$ GPLGSPEF $^{80}$ ) which were left from the linker after proteolytically removing the N-terminal GST tag and differed from the native sequence in the corresponding region. Consistently, we observed a tetramer population of TaNAC in a solution study (Supplemental Fig. 1E). Similar to the $\mathrm{NAC}_{\alpha}-\mathrm{NAC}_{\beta}$ heterodimer, the two NAC domains of TaNAC homodimer were related by a pseudo dyad symmetry $\left(178^{\circ}\right.$ rotation and $0.24 \AA$ screw length in $\mathrm{AB}$; and $179^{\circ}$ rotation and $0.36 \AA$ screw length in $\mathrm{CD}$ ). Furthermore, the two homodimer were related by $175^{\circ}$ rotation angle and $0.12 \AA$ screw length. The dimerization buried total of $1980 \AA^{2}$ and $2050 \AA^{2}$ solvent accessible surface in each of the two TaNAC homodimers, respectively. Formation of the tetramer further buried $2580 \AA^{2}$ solvent accessible surface. Comparing with corresponding native residues of aNAC (i.e., ${ }^{73} \mathrm{EKKARKAM}^{80}$ ), we found that if we use native residues $\left({ }^{78} K A M^{80}\right)$ to replace the corresponding non-native residues $\left({ }^{78} \mathrm{PEF}^{80}\right)$, there would be little influence on the backbone of TaNAC (e.g., their $\varphi-\phi$ angles). However, the rest residue substitution would disturb the local structure because of side chain collisions. Therefore, we concluded that the observed tetramer of TaNAC is more likely to be an artifact of crystal packing, and it probably has no physiologic significance.

\section{Highly conserved NAC domain from Archaea to Human}

To identify potential homologous structures of our $\mathrm{NAC}_{\alpha}-$ $\mathrm{NAC}_{\beta}$ heterodimer, we compared our crystal structure with those in the Protein Data Bank (PDB) using the Dali search engine (Holm and Sander, 1993). The most similar folding found from this search was, as expected, the NAC domain homodimer from the archaea, Methanothermobacter marburgensis (Spreter et al., 2005), and no other significant hit emerged. Further analyses showed that this NAC domain of aeNAC is similar to our structures of $\mathrm{NAC}_{\alpha}(1.0 \AA \mathrm{rmsd}$ for 42 Ca pairs), $\mathrm{NAC}_{\beta}$ (0.86 $\AA$ rmsd for $42 \mathrm{Ca}$ pairs), and TaNAC (0.85 $\AA$ rmsd for $44 \mathrm{Ca}$ pairs) at the protomer level (Fig. $2 \mathrm{~A})$ and to $\mathrm{NAC}_{\alpha}-\mathrm{NAC}_{\beta}(0.93 \AA$ rmsd for $83 \mathrm{C \alpha}$ pairs $)$ and TaNACTaNAC $(0.85 \mathrm{rmsd} \AA$ for $87 \mathrm{Ca}$ pairs) at the dimer level (Fig. 2B).

\section{DISCUSSION}

Eukaryotic NAC is an important protein complex functioning as a stable heterodimer of aNAC and BNAC subunits in cotranslation targeting (Lauring et al., 1995a, b; Powers and Walter, 1996; Moller et al., 1998b) and X-junction related pathways (Whitby and Dixon, 2001) among others. The crystal structure of homologous aeNAC from archaea, which has only one NAC-like gene, shows that aeNAC forms a homodimer through the NAC domains (Spreter et al., 2005). Deletion of the C-terminal UBA domain in human aNAC does not influence the NAC activity in binding nascent polypeptide ribosome complex (Spreter et al., 2005). Therefore, based on sequence homology it is anticipated that the dimerization of NAC was mainly mediated by the NAC domains of $\alpha$ NAC and BNAC subunits.

To study the three-dimensional structure of NAC, we coexpressed recombinant proteins of the two subunits of human NAC, aNAC and $\beta N A C$, in E. coli. The coexpression overcame problems of low yield associated with individually expressed proteins and eliminated the step of reconstitution of NAC from its recombinant components (Beatrix et al., 2000). Interestingly, although we set up the crystallization experiment with full length NAC heterodimer, the final crystal structure contained only the NAC domains after six-month crystallization. It suggests that other regions of the two subunits are more flexible and sensitive to degradation. The crystal structure of the heterodimer of the NAC domains of $\mathrm{NAC}_{\alpha}$ and $\mathrm{NAC}_{\beta}$ indeed has a similar folding and dimer formation to the previously reported aeNAC dimer. It is clear that hydrogen bonding in two joint $\beta$-sheets and a hydrophobic core between the two sheets are the main driving force in dimerization. Therefore, an isolated NAC subunit of either $\mathrm{NAC}_{\alpha}$ or $\mathrm{NAC}_{\beta}$ is unlikely to stay as stable, soluble monomers in solution because of unsaturated hydrogen bonding potential and extensively exposed hydrophobic surface.

Structure based sequence alignment shows that there are ten pairs of identical residues between the observed structures of the two subunits (Fig. 1C). Among them, Gly90, Pro109, Gly124 in $\mathrm{NAC}_{\alpha}$ and corresponding Gly58', Pro77', and Gly92' in $\mathrm{NAC}_{\beta}$ were located at the bottom of $\mathrm{V}$ shaped structure, connecting each of the three pairs of $\beta$ strands, $\beta 1-\beta 2, \beta 3-\beta 4$, and $\beta 5-\beta 6$ from the two arms. The special main chain properties of these conserved glycine and proline residues likely contribute to the correct folding of the subunits. This notion is further supported by an observation that these residues are also conserved in aNAC homologs from different species (Fig. 3). Some of the remaining conserved residues may contribute to formation of the hydrophobic core of the NAC domain dimer.

Isolated aNAC subunits play several important roles distinct from those of the NAC complex. For example, aNAC can function as a transcription coactivator (Moreau et al., 1998; St-Arnaud, 1998; St-Arnaud and Quelo, 1998; Yotov et al., 1998); it is a positive regulator in human 
erythroid-cell differentiation (Lopez et al., 2005) and a negative regulator in the proliferation, differentiation, and cytotoxic activation of CD8 (+) T cells (Al-Shanti et al., 2004; Al-Shanti and Aldahoodi, 2006). Furthermore, aNAC can interact with several disease related proteins (Goatley et al., 2002; Mossabeb et al., 2002; Li et al., 2005; Mittermann et al., 2008). What does isolated aNAC look like? And how does it function in the absence of $\beta N A C$ ? To address these questions, we expressed recombinant proteins of a full length aNAC and its NAC domain (TaNAC). Solution studies on both aNAC and TaNAC showed that they all exist in solution as stable homodimers, suggesting that the homodimer of full length aNAC shares the same interface with the TaNAC homodimer. More importantly our crystal structure of the
TaNAC homodimer shows a very similar folding and dimerization to the $\mathrm{NAC}_{\alpha}-\mathrm{NAC}_{\beta}$ heterodimer as well as the previously reported aeNAC homodimer (Fig. 2).

Our solution study showed that full length $\beta N A C$ can form a homodimer (Supplemental Fig. 4). It is interesting to ask whether $B N A C$ also forms the homodimer via its NAC domain. In this context, we made a model of a homodimer of $B N A C$ NAC domain (TßNAC, Supplemental Fig. 3) using the SWISS-MODEL online server (Guex and Peitsch, 1997; Schwede et al., 2003; Arnold et al., 2006) and the $\mathrm{NAC}_{\alpha^{-}}$ $\mathrm{NAC}_{\beta}$ heterodimer as the template structure. We assessed packing quality of this TBNAC model using the atomic empirical mean force potential implemented in the program ANOLEA (Melo and Feytmans, 1998) and using the program

$\quad$ A
hNACA
Mouse_NACA
D.m_NACA
S.c_Egd2
aeNAC

hNACA

hNACA Mouse_NACA D.m_NACA S.cEgd2 aeNAC
1 MPG...EATETVPATEQEL.PQPQAETGSGTESDSDESVPELEEQDST QATTQQAQLAAAAEIDEEPVSKAKQSRSEKKARK 1 MPG...EATETVPATEQEL.PQPQAETGSGTESDSDESVPELEEQDSTQTATQQAQLAAAAEIDEEPVSKAKQSRSEKKARK 1 MPELTEIKSEAAPSTSAEAKPEDVRVEDDGSDSDSDGGMPGLEE. . AVAATTQLGGGTGLPID. LVSKAKQSRGEKKARK

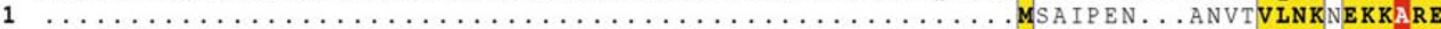

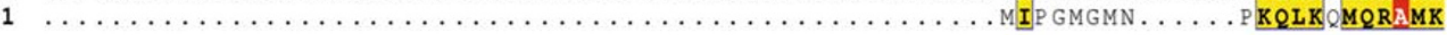

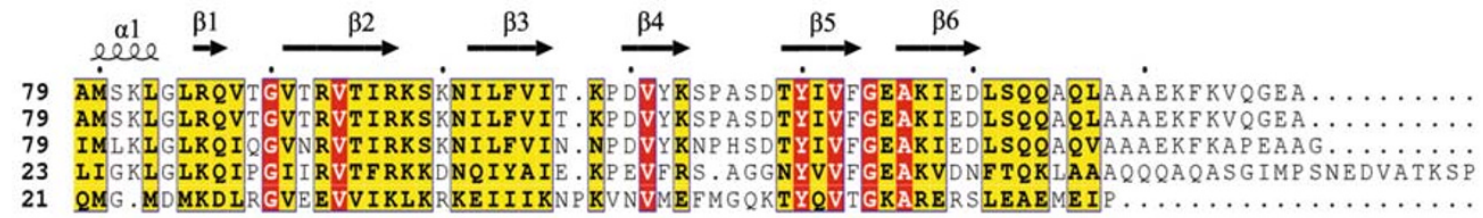

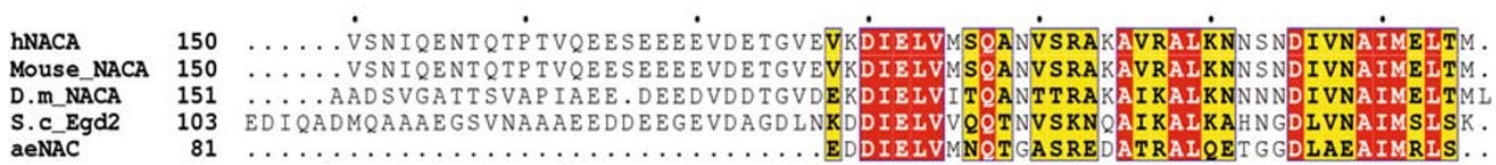

B

h_BTF3b
mouse_BTF3
D.m_BTF3
S.c_BTT1
S.c_egd1p

h_BTF3b h_BTF $3 b$ mouse_BTF 3

D. $m \_B T F 3$ S. C_BTT1

s.c_egdip
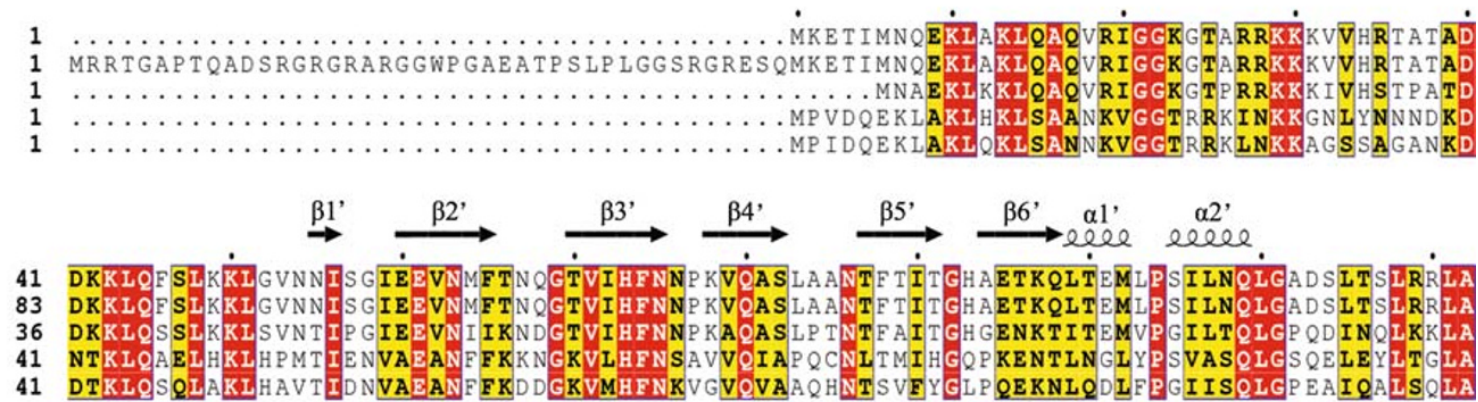

h_BTF $3 b$ mouse_BTF D. $\mathrm{m}$ BTF 3 S. CBTT1 S.c_egdip

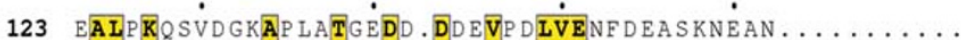

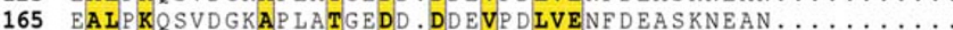
118 TEIASKSGAGGA A SSAADA GDDDVP DLVENFEEVAIADTKEEKSGEVAASA

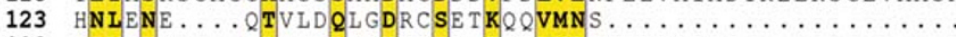

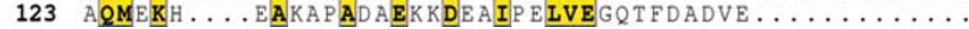

Figure 3. Multiple sequence alignment of NAC homologs from human to archaea. NAC $\alpha-N^{\prime} C_{\beta}$ secondary structure elements are also labeled. (A) Alignment of aNAC homologs: hNACA, human aNAC, GenBank ID AAX14393.1;mouse_NACA, mouse, AAH99375.1; D.m_NACA, fruit fly, AAM68653.1; S.C_Egd2, yeast, AAA92080.1; and aeNAC, archaea, NP_275320. (B) Alignment of $\beta N A C$ homologs: h_BTF3b, human BNAC, AAH08062.1; mouse_BTF3, mouse, AAH64010.1; D.m_BTF3, fruit fly, AAF06076.1; S.c_BTT1, yeast, CAA55370; and S.c_egd1p, yeast, CAA55371.1. 
PROCHECK (Laskowski et al., 2005). The overall stereochemical quality was good, and energy environment for majority residues were favorable (data not shown). It suggests that BNAC can form a homodimer in a manner similar to the NAC heterodimer as well as the aNAC homodimer.

With our current constructs, the recombinant proteins of full length aNAC (23.3 kDa) and $\beta N A C(17.7 \mathrm{kDa})$ and different species of their combinations could be distinguished with a number of techniques based on molecular weight. During the purification process, we observed a stable heterodimer of aNAC and $\beta N A C$ with a 1:1 stoichiometry of the two subunits as reported previously (Beatrix et al., 2000), which migrated as a single macromolecule, but no other combinations of the two species appeared (Supplemental Fig. 1A). Meanwhile, individually purified aNAC, $\beta N A C$ or TaNAC formed stable homodimers in a number of tested solutions shown in size chromatography and analytical ultracentrifugation (Supplemental Fig. 1B-E). These observations raise a question: Why does the heterodimer become the dominant species when aNAC and BNAC co-exist? In other words, why is the NAC heterodimer complex more stable than the aNAC or BNAC homodimer?

A significant difference between the heterodimer and homodimer comes from their interface charge distribution as shown in Fig. 4. The pl values of $\mathrm{NAC}_{\alpha}$ and $\mathrm{NAC}_{\beta}$ are 9.8 and 5.5 , respectively (estimated using the program DNASTAR (Madison, WI)). These two subunits carry opposite electric charges under neutral $\mathrm{pH}$. Thus, on top of other favorable intermolecular interactions the electrostatic interaction contributes favorably to the overall binding energy between $N A C_{\alpha}-N A C_{\beta}$. In contrast, in a homodimer both TaNAC subunits, for example, carry positive charges, and the electrostatic term of the binding energy between the two subunits in a TaNAC homodimer is unfavorable, although it may not cancel completely other favorable terms in the total binding energy. In particular, on the interface of $\mathrm{NAC}_{\alpha}-\mathrm{NAC}_{\beta}$ heterodimer, Arg93, Arg97, and Asp118 of $\mathrm{NAC}_{\alpha}$ are close to the lle110' carboxyl oxygen, Glu60' (and Glu61') side chain, and His93' side chain of $\mathrm{NAC}_{\beta}$, respectively, thus providing favorable binding energy. In contrast, on the interface of TaNAC homodimer, Arg93 and Arg97 of subunit A are close to Arg97 and Arg93 from subunit B, thus contributing negatively to the dimerization. Therefore, electrostatic interaction is likely to play a key role in stability difference between the TaNAC homodimer and $\mathrm{NAC}_{\alpha}-\mathrm{NAC}_{\beta}$ heterodimer.

To investigate the generality of our crystal structures, we aligned amino acid sequences of homologs of aNAC and BNAC from archaea (Methanothermobacter marburgensis), yeast (Saccharomyces cerevisiae), fruit fly (Drosophila melanogaster), mouse (Mus musculus), and humans (Homo sapiens). As reported before (Spreter et al., 2005), aNAC homologs share two conserved domains. One is the NAC domain which is observed in our crystal structures, and the other is a UBA (ubiquitin-associated) domain which would be C-terminal to the NAC domain but was lost during crystallization (Fig. 3A). Similarly, BNAC homologs share two conserved regions. One is the NAC domain, and the other is N-terminal to the NAC domain (Fig. 3B). The latter conserved region is potentially responsible for conserved functions such as ribosome binding (Wegrzyn et al., 2006). It is quite probable that an ancestor gene coding a NAC-like domain was duplicated into two copies. Subsequently, one evolved to aNAC homologs, and the other to BNAC homologs. They all preserved the NAC domain presumably to maintain the dimerization ability and other NAC associated functions while allowing new functions to be developed by additional structural features.

In summary, we determined two crystal structures of $\mathrm{NAC}_{\alpha^{-}}$ $N_{A C}$ and TaNAC at $1.9 \AA$ and $2.4 \AA$ resolutions, respectively. Different NAC domains show very similar folding at both protomer and dimer levels despite of the low sequence identify between aNAC and BNAC subunits. Analyses on the charge distributions suggest that electrostatic interaction between subunits contribute to the stability difference between the NAC heterodimer and aNAC homodimer. However, may there be any other function in the NAC domain of NAC subunits in additional to dimerization? Are the positively charged surface residues of $\mathrm{NAC}_{\alpha}$ related to nucleic acid binding of aNAC? Such questions warrant further investigation.

\section{MATERIALS AND METHODS}

\section{Expression and purification of the human NAC complex and the NAC domain of aNAC}

The coding sequences of full length human aNAC and BNAC were amplified by PCR from an in-house human cDNA library and inserted between the EcoRI and Sall restriction sites and between the Ndel and $X$ hol restriction sites of the pETDuet- 1 coexpression vector (Novagen), respectively. The recombinant $\mathrm{His}_{6}-\mathrm{aNAC}$ and $\beta N A C$ were coexpressed in E. coli strain BL21 (DE3). The cell culture was induced at $0.6 \mathrm{OD}_{600}$ with $0.5 \mathrm{mM}$ Isopropyl-beta-D-thiogalactopyranoside (IPTG). The cells were disrupted with a French Press instrument (Sim-Aminco) at 15,000 psi in a lysis buffer of $20 \mathrm{mM}$ Tris$\mathrm{HCl}(\mathrm{pH} 8.0), 500 \mathrm{mM} \mathrm{NaCl}$, and $5 \%(\mathrm{v} / \mathrm{v})$ glycerol. The lysate was centrifuged at $30,000 \mathrm{~g}$ for $30 \mathrm{~min}$. The supernatant after centrifugation was loaded onto a Ni affinity column (GE Healthcare), followed by washing with 20 column volume of the lysis buffer. $\mathrm{His}_{6}-\alpha \mathrm{NAC}$ and BNAC were co-eluted with $200 \mathrm{mM}$ imidazole in the lysis buffer. Recombinant NAC was further purified using sequential HiTrap Heparin HP, ion-exchange Mono Q, and gel-filtration Superdex 200 10/300 GL (GE Healthcare) chromatography. The purified NAC from Superdex 200 was concentrated to $33 \mathrm{mg} / \mathrm{mL}$ (BCA Protein Assay Kit, GeneStar Biosolutions Co., Ltd.) in a storage buffer of $20 \mathrm{mM}$ Tris$\mathrm{HCl}(\mathrm{pH} \mathrm{8.0)}$ and $350 \mathrm{mM} \mathrm{NaCl}$.

The DNA sequence encoding for the NAC domain of aNAC (i.e., TaNAC, residues 80-133 based on the best refinement result at the time) was subcloned into the pGEX-6P-1 vector (Amersham 

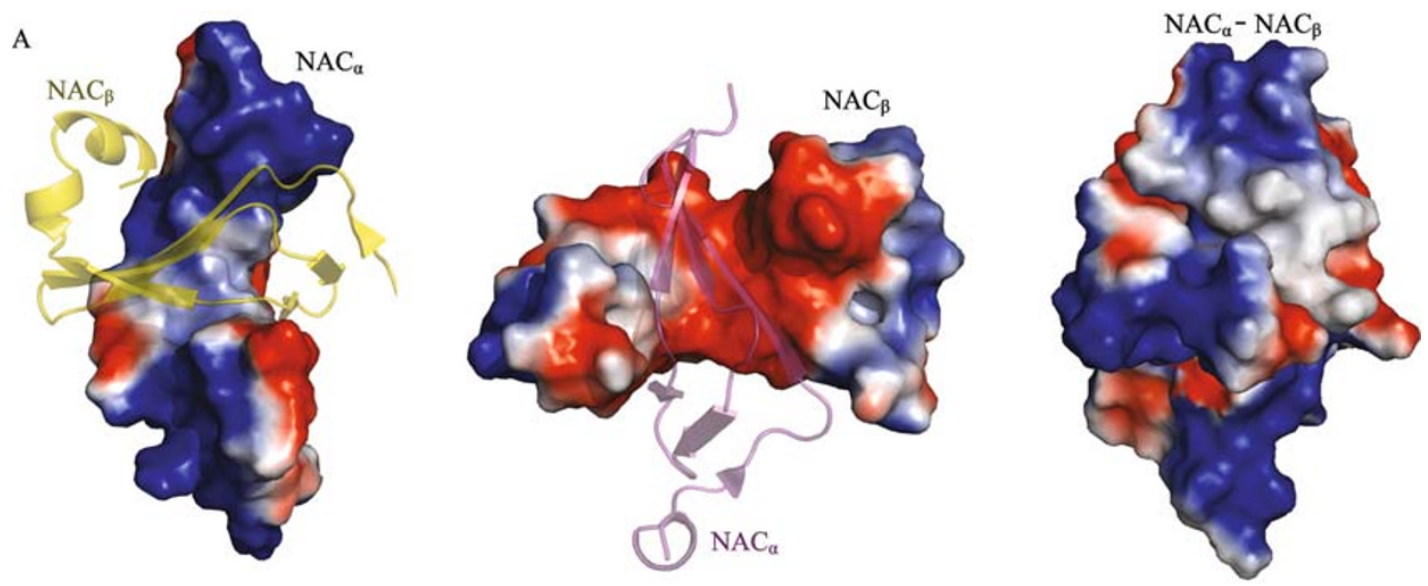

B
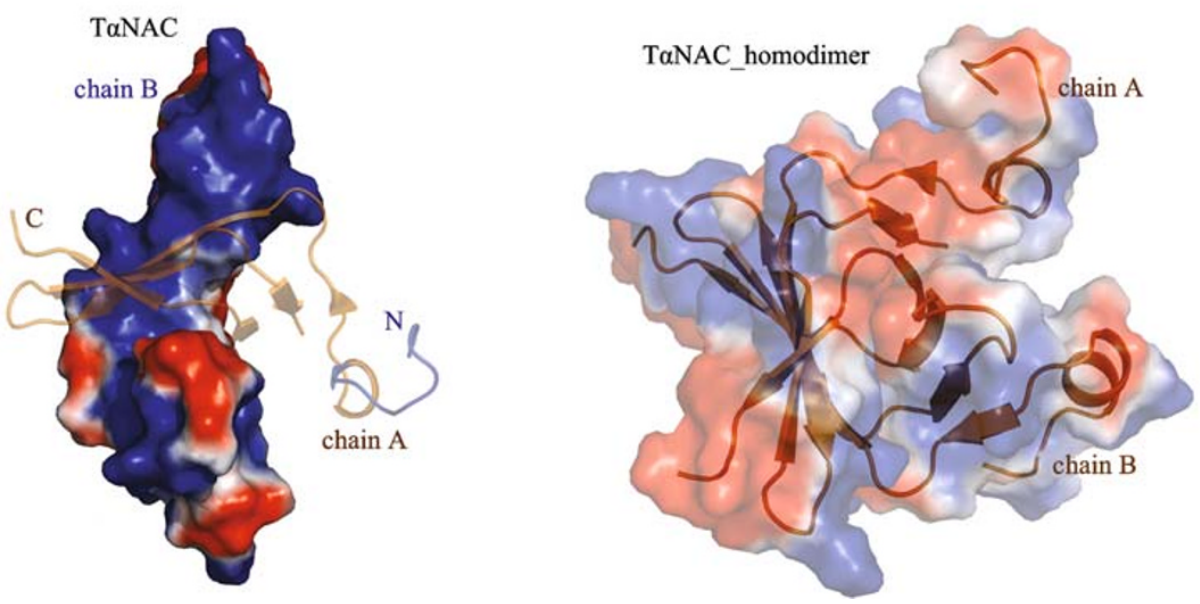

Figure 4. Different binding interfaces of $\mathrm{NAC}_{\alpha}-\mathrm{NAC}_{\beta}$ heterodimer and TaNAC homodimer. In the surface presentation, blue, red, and white colors represent positively charged region, negatively charged region, and neutral zone, respectively. (A) Surface presentation of electrostatic potential of the binding surface between $\mathrm{NAC}_{\alpha}$ and $\mathrm{NAC}_{\beta}$. On the left side and in the middle, the corresponding binding partner is shown in a ribbon presentation. The right side panel shows the dimer surface model. (B) Surface representation of TaNAC protomer. The left panel has a similar orientation to that of $\mathrm{NAC}_{\alpha}$ in panel $\mathrm{A}$. The right panel shows a semitransparent surface model of the homodimer superimposed with its ribbon presentation.

Biosciences) between EcoRl and Sall restriction sites. Recombinant fusion protein with an N-terminal glutathione S-transferase (GST) was expressed in $E$. coli strain BL21 (DE3) and extracted using glutathione sepharose affinity column (Amersham Biosciences) in the same lysis buffer as above. TaNAC was proteolytically released from GST with PreScission Protease (Phamacia Biotech) and was further purified by sequential chromatography including HiTrap Heparin HP and Superdex 75 10/300 GL (GE Healthcare). The purified TaNAC sample was concentrated to $8.3 \mathrm{mg} / \mathrm{mL}$, assayed as above.

\section{Crystallization and structure determination}

Crystals of the NAC complex were grown at $16^{\circ} \mathrm{C}$ using the hanging drop vapor diffusion method. The protein solution was mixed in a $1 \mu \mathrm{L}$ $+1 \mu \mathrm{L}$ format with reservoir solution $(500 \mu \mathrm{L})$. Crystal screening was carried out using Hampton Research crystal screening kits. Crystals of good diffraction quality appeared in $0.1 \mathrm{M} \mathrm{NaCl}, 0.1 \mathrm{M}$ sodium citrate tribasic dehydrate $(\mathrm{pH} 5.6)$, and $12 \%(\mathrm{w} / \mathrm{V})$ polyethylene glycol
(PEG) 4000 (i.e., No. 17 of $\mathrm{MembFac}^{\mathrm{TM}}$, named as mother liquid $\mathrm{A}$ hereafter) after long time incubation (about 6 months). They were cryoprotected by being quickly dipped into a cryoprotectant solution (i.e., mother liquid A supplemented with $12.5 \%$ ( $v / v$ ) glycerol). lodide derivatives were prepared by soaking native crystals in the mother liquid $\mathrm{A}$ plus $0.75 \mathrm{M} \mathrm{KI}$ for $2 \mathrm{~min}$ and then $1 \mathrm{M} \mathrm{KI}$ for $2 \mathrm{~min}$.

TaNAC crystals grew more quickly than the NAC complex, despite of following the same crystallization protocol. It took only 3-4d for high diffraction-quality crystals to grow up in mother liquid $B(0.2 \mathrm{M}$ potassium citrate tribasic monohydrate $(\mathrm{pH} 8.3)$ and $20 \%(w / v)$ PEG 3350). Heavy atom derivative crystals were prepared similarly to the above, except that TaNAC crystals were soaked only $1 \mathrm{~min}$ in mother liquid $\mathrm{B}$ plus $0.4 \mathrm{M} \mathrm{KI}$.

Crystals were screened for good diffraction from native and heavy atom derivatives with an in-house FR-E VariMax X-ray source (Rigaku). High resolution data sets were collected at the beam line 17A of KEK Photon Factory, Japan. All data sets were processed using the program HKL2000 (Otwinowski and Minor, 1997). Phases of the crystal structures of both NAC complex and $\alpha$ NAC NAC domain 
were determined using SAD method and the Phenix software suite (Adams et al., 2010) (including Phenix.autosol and Phenix.autobuild) and the CCP4 program package (Potterton et al., 2003). Manual model building was carried out using the Coot program (Emsley and Cowtan, 2004), and refinement alternated between cycles using the Phenix software suite and Refmac5 (Murshudov et al., 1997). Data collection, phasing, and refinement statistics are summarized in Table 1. Secondary structures were defined using the DSSP program (Kabsch and Sander, 1983). Structural analyses including superposition of three dimensional structures, calculation of rotation angles, screw lengths, and rmsd, and analyzing dimerization interface were carried out using the program EdPDB (Zhang and Matthews, 1995). Structural ribbon diagrams and molecular surface diagrams were prepared with the program PyMol (Delano, 2002). Sequence alignment was performed using the program ClustaX2.0 (Larkin et al., 2007), and the alignment figures were generated using the online ESPript server (Gouet et al., 1999).

\section{COORDINATE DEPOSITION}

Coordinates of the refined models of $\mathrm{NAC}_{\alpha}-\mathrm{NAC}_{\beta}$ complex and TaNAC and their experimental structural factors have been deposited to the RCSB Protein Data Bank (http://www.rcsb.org/pdb/). The accession ID is $3 \mathrm{MCB}$ and $3 \mathrm{MCE}$, respectively.

\section{ACKNOWLEDGEMENTS}

We are grateful to staff members of the Structural Biology Core Facility in the Institute of Biophysics, CAS, for their excellent technical assistance, especially Mr. Yi Han for his help in data collection, Ms. Xiaoxia Yu for her assistance in analytical ultracentrifugation, and $\mathrm{Mr}$. Xudong Zhao for his technical support. This work was supported by the National Natural Science Foundation of China (grant No. 30730022), the National Basic Research Program (973 Program) (grant Nos. 2006CB806503 and 2007CB914304), the National Programs for High Technology Research and Development Program (863 Program) (grant Nos. 2006AA02A322 and 2006AA020502), and the CAS (China) grant KSCX2-YW-R-05 to Z.R.

\section{ABBREVIATIONS}

aNAC, alpha subunit of NAC; $\beta N A C$, beta subunit of NAC; ER, endoplasm reticulum; GST, glutathione S-transferase; IPTG, Isopropyl-beta-D-thiogalactopyranoside; NAC, nascent polypeptide-associated complex; PEG, polyethylene glycol; rmsd, root mean square deviation; SAD, single wavelength anomalous diffraction; SRP, signal recognition particle; TaNAC, truncated aNAC; UBA domain, ubiquitin-associated domain

\section{REFERENCES}

Adams, P.D., Afonine, P.V., Bunkoczi, G., Chen, V.B., Davis, I.W., Echols, N., Headd, J.J., Hung, L.W., Kapral, G.J., GrosseKunstleve, R.W., et al. (2010). PHENIX: a comprehensive Python-based system for macromolecular structure solution. Acta Crystallogr D Biol Crystallogr 66, 213-221.

Al-Shanti, N., and Aldahoodi, Z. (2006). Inhibition of alpha nascent polypeptide associated complex protein may induce proliferation, differentiation and enhance the cytotoxic activity of human CD8 + T cells. J Clin Immunol 26, 457-464.

Al-Shanti, N., Steward, C.G., Garland, R.J., and Rowbottom, A.W. (2004). Investigation of alpha nascent polypeptide-associated complex functions in a human CD8(+) T cell ex vivo expansion model using antisense oligonucleotides. Immunology 112, 397-403.

Arnold, K., Bordoli, L., Kopp, J., and Schwede, T. (2006). The SWISSMODEL workspace: a web-based environment for protein structure homology modelling. Bioinformatics 22, 195-201.

Beatrix, B., Sakai, H., and Wiedmann, M. (2000). The alpha and beta subunit of the nascent polypeptide-associated complex have distinct functions. J Biol Chem 275, 37838-37845.

Delano, W. (2002). The PyMOL Molecular Graphics System. http:// www.pymol.org.

Deng, J.M., and Behringer, R.R. (1995). An insertional mutation in the BTF3 transcription factor gene leads to an early postimplantation lethality in mice. Transgenic Res 4, 264-269.

Emsley, P., and Cowtan, K. (2004). Coot: model-building tools for molecular graphics. Acta Crystallogr D Biol Crystallogr 60, 2126-2132.

Fünfschilling, U., and Rospert, S. (1999). Nascent polypeptideassociated complex stimulates protein import into yeast mitochondria. Mol Biol Cell 10, 3289-3299.

George, R., Beddoe, T., Landl, K., and Lithgow, T. (1998). The yeast nascent polypeptide-associated complex initiates protein targeting to mitochondria in vivo. Proc Natl Acad Sci U S A 95, 2296-2301.

Goatley, L.C., Twigg, S.R., Miskin, J.E., Monaghan, P., St-Arnaud, R., Smith, G.L., and Dixon, L.K. (2002). The African swine fever virus protein $\mathrm{j} 4 \mathrm{R}$ binds to the alpha chain of nascent polypeptideassociated complex. J Virol 76, 9991-9999.

Gouet, P., Courcelle, E., Stuart, D.I., and Métoz, F. (1999). ESPript: analysis of multiple sequence alignments in PostScript. Bioinformatics $15,305-308$.

Guex, N., and Peitsch, M.C. (1997). SWISS-MODEL and the SwissPdbViewer: an environment for comparative protein modeling. Electrophoresis 18, 2714-2723.

Holm, L., and Sander, C. (1993). Protein structure comparison by alignment of distance matrices. J Mol Biol 233, 123-138.

Kabsch, W., and Sander, C. (1983). Dictionary of protein secondary structure: pattern recognition of hydrogen-bonded and geometrical features. Biopolymers 22, 2577-2637.

Kim, S.H., Shim, K.S., and Lubec, G. (2002). Human brain nascent polypeptide-associated complex alpha subunit is decreased in patients with Alzheimer's disease and Down syndrome. J Investig Med 50, 293-301.

Kroes, R.A., Jastrow, A., McLone, M.G., Yamamoto, H., Colley, P., Kersey, D.S., Yong, V.W., Mkrdichian, E., Cerullo, L., Leestma, J., et al. (2000). The identification of novel therapeutic targets for the treatment of malignant brain tumors. Cancer Lett 156, 191-198.

Larkin, M.A., Blackshields, G., Brown, N.P., Chenna, R., McGettigan, P.A., McWilliam, H., Valentin, F., Wallace, I.M., Wilm, A., Lopez, R., et al. (2007). Clustal $W$ and Clustal $X$ version 2.0. Bioinformatics 23, 2947-2948.

Laskowski, R.A., Chistyakov, V.V., and Thornton, J.M. (2005). PDBsum more: new summaries and analyses of the known 3D structures of proteins and nucleic acids. Nucleic Acids Res 33, D266-D268.

Lauring, B., Kreibich, G., and Weidmann, M. (1995a). The intrinsic 
ability of ribosomes to bind to endoplasmic reticulum membranes is regulated by signal recognition particle and nascent-polypeptideassociated complex. Proc Natl Acad Sci U S A 92, 9435-9439.

Lauring, B., Sakai, H., Kreibich, G., and Wiedmann, M. (1995b). Nascent polypeptide-associated complex protein prevents mistargeting of nascent chains to the endoplasmic reticulum. Proc Natl Acad Sci U S A 92, 5411-5415.

Li, D., Wang, X.Z., Ding, J., and Yu, J.P. (2005). NACA as a potential cellular target of hepatitis B virus preS1 protein. Dig Dis Sci 50 , 1156-1160.

Lopez, S., Stuhl, L., Fichelson, S., Dubart-Kupperschmitt, A., St Arnaud, R., Galindo, J.R., Murati, A., Berda, N., Dubreuil, P., and Gomez, S. (2005). NACA is a positive regulator of human erythroid-cell differentiation. J Cell Sci 118, 1595-1605.

Markesich, D.C., Gajewski, K.M., Nazimiec, M.E., and Beckingham, K. (2000). Bicaudal encodes the Drosophila beta NAC homolog, a component of the ribosomal translational machinery*. Development 127, 559-572.

Melo, F., and Feytmans, E. (1998). Assessing protein structures with a non-local atomic interaction energy. J Mol Biol 277, 1141-1152.

Mittermann, I., Reininger, R., Zimmermann, M., Gangl, K., Reisinger, J., Aichberger, K.J., Greisenegger, E.K., Niederberger, V., Seipelt, J., Bohle, B., et al. (2008). The IgE-reactive autoantigen Hom s 2 induces damage of respiratory epithelial cells and keratinocytes via induction of IFN-gamma. J Invest Dermatol 128, 1451-1459.

Moller, I., Beatrix, B., Kreibich, G., Sakai, H., Lauring, B., and Wiedmann, M. (1998a). Unregulated exposure of the ribosomal Msite caused by NAC depletion results in delivery of non-secretory polypeptides to the Sec61 complex. FEBS Lett 441, 1-5.

Moller, I., Jung, M., Beatrix, B., Levy, R., Kreibich, G., Zimmermann, R., Wiedmann, M., and Lauring, B. (1998b). A general mechanism for regulation of access to the translocon: competition for a membrane attachment site on ribosomes. Proc Natl Acad Sci U S A 95, 13425-13430.

Moreau, A., Yotov, W.V., Glorieux, F.H., and St-Arnaud, R. (1998). Bone-specific expression of the alpha chain of the nascent polypeptide-associated complex, a coactivator potentiating cJun-mediated transcription. Mol Cell Biol 18, 1312-1321.

Mossabeb, R., Seiberler, S., Mittermann, I., Reininger, R., Spitzauer, S., Natter, S., Verdino, P., Keller, W., Kraft, D., and Valenta, R. (2002). Characterization of a novel isoform of alpha-nascent polypeptide-associated complex as IgE-defined autoantigen. J Invest Dermatol 119, 820-829.

Murshudov, G.N., Vagin, A.A., and Dodson, E.J. (1997). Refinement of macromolecular structures by the maximum-likelihood method. Acta Crystallogr D Biol Crystallogr 53, 240-255.

Otwinowski, Z., Minor, W. (1997). Processing of X-ray diffraction data collected in oscillation mode. Methods Enzymol 276, 307-326.

Panasenko, O., Landrieux, E., Feuermann, M., Finka, A., Paquet, N., and Collart, M.A. (2006). The yeast Ccr4-Not complex controls ubiquitination of the nascent-associated polypeptide (NAC-EGD) complex. J Biol Chem 281, 31389-31398.

Panasenko, O.O., David, F.P., and Collart, M.A. (2009). Ribosome association and stability of the nascent polypeptide-associated complex is dependent upon its own ubiquitination. Genetics 181, 447-460.

Potterton, E., Briggs, P., Turkenburg, M., and Dodson, E. (2003). A graphical user interface to the CCP4 program suite. Acta Crystal- logr D Biol Crystallogr 59, 1131-1137.

Powers, T., and Walter, P. (1996). The nascent polypeptideassociated complex modulates interactions between the signal recognition particle and the ribosome. Curr Biol 6, 331-338.

Reimann, B., Bradsher, J., Franke, J., Hartmann, E., Wiedmann, M., Prehn, S., and Wiedmann, B. (1999). Initial characterization of the nascent polypeptide-associated complex in yeast. Yeast 15 , 397-407.

Scheuring, U.J., Corbeil, J., Mosier, D.E., and Theofilopoulos, A.N. (1998). Early modification of host cell gene expression induced by HIV-1. AIDS 12, 563-570.

Schwede, T., Kopp, J., Guex, N., and Peitsch, M.C. (2003). SWISSMODEL: An automated protein homology-modeling server. Nucleic Acids Res 31, 3381-3385.

Shi, X., Parthun, M.R., and Jaehning, J.A. (1995). The yeast EGD2 gene encodes a homologue of the alpha NAC subunit of the human nascent-polypeptide-associated complex. Gene 165, 199-202.

Spreter, T., Pech, M., and Beatrix, B. (2005). The crystal structure of archaeal nascent polypeptide-associated complex (NAC) reveals a unique fold and the presence of a ubiquitin-associated domain. $\mathrm{J}$ Biol Chem 280, 15849-15854.

St-Arnaud, R. (1998). Transcriptional regulation during mesenchymal cell differentiation: the role of coactivators. Crit Rev Eukaryot Gene Expr 8, 191-202.

St-Arnaud, R., and Quelo, I. (1998). Transcriptional coactivators potentiating AP-1 function in bone. Front Biosci 3, d838-d848.

Thiede, B., Dimmler, C., Siejak, F., and Rudel, T. (2001). Predominant identification of RNA-binding proteins in Fas-induced apoptosis by proteome analysis. J Biol Chem 276, 26044-26050.

Wang, S., Sakai, H., and Wiedmann, M. (1995). NAC covers ribosome-associated nascent chains thereby forming a protective environment for regions of nascent chains just emerging from the peptidyl transferase center. J Cell Biol 130, 519-528.

Wegrzyn, R.D., Hofmann, D., Merz, F., Nikolay, R., Rauch, T., Graf, C., and Deuerling, E. (2006). A conserved motif is prerequisite for the interaction of NAC with ribosomal protein L23 and nascent chains. J Biol Chem 281, 2847-2857.

Whitby, M.C., and Dixon, J. (2001). Fission yeast nascent polypeptide-associated complex binds to four-way DNA junctions. J Mol Biol 306, 703-716.

Wiedmann, B., Sakai, H., Davis, T.A., and Wiedmann, M. (1994). A protein complex required for signal-sequence-specific sorting and translocation. Nature 370, 434-440.

Yotov, W.V., Moreau, A., and St-Arnaud, R. (1998). The alpha chain of the nascent polypeptide-associated complex functions as a transcriptional coactivator. Mol Cell Biol 18, 1303-1311.

Zhang, X.-J., and Matthews, B.W. (1995). EDPDB: a multifunctional tool for protein structure analysis. J Appl Cryst 28, 624-630.

Zheng, X.M., Moncollin, V., Egly, J.M., and Chambon, P. (1987). A general transcription factor forms a stable complex with RNA polymerase B (II). Cell 50, 361-368.

Zheng, X.M., Black, D., Chambon, P., and Egly, J.M. (1990). Sequencing and expression of complementary DNA for the general transcription factor BTF3. Nature 344, 556-559.

Zuo, L., Ogle, C.K., Fischer, J.E., and Nussbaum, M.S. (1997). mRNA differential display of colonic mucosa cells in ulcerative colitis. J Surg Res 69, 119-127. 